\title{
Determinants of Exclusive Breastfeeding Cessation in the Early Postnatal Period among Culturally and Linguistically Diverse (CALD) Australian Mothers
}

\author{
Felix Akpojene Ogbo ${ }^{1,2, *}$, Osita Kingsley Ezeh ${ }^{3}{ }^{\circledR}$, Sarah Khanlari ${ }^{4,5}{ }^{\circledR}$, Sabrina Naz ${ }^{1}$, \\ Praween Senanayake ${ }^{1}$, Kedir Y. Ahmed ${ }^{1}{ }^{1}$, Anne McKenzie ${ }^{6}$, Olayide Ogunsiji ${ }^{7}$, \\ Kingsley Agho ${ }^{1,3}$, Andrew Page ${ }^{1}$, Jane Ussher ${ }^{1}$, Janette Perz ${ }^{1}$, Bryanne Barnett AM $^{8}$ and \\ John Eastwood 4,9,10,11,12,13 \\ 1 Translational Health Research Institute, School of Medicine, Western Sydney University, \\ Campbelltown Campus, Locked Bag 1797, Penrith, NSW 2571, Australia \\ 2 General Practice Unit, Prescot Specialist Medical Centre, Welfare Quarters, Makurdi, \\ Benue State 972261 , Nigeria \\ 3 School of Science and Health, Western Sydney University, Campbelltown Campus, Locked Bag 1797, Penrith, \\ NSW 2571, Australia \\ 4 Department of Community Paediatrics, Sydney Local Health District, Croydon Community Health Centre, \\ 24 Liverpool Street, Croydon, NSW 2132, Australia \\ 5 School of Medicine and Public Health, University of Newcastle, University Drive, Callaghan, \\ NSW 2308, Australia \\ 6 Child and Family Health Nursing, Primary \& Community Health, South Western Sydney Local Health \\ District, Narellan, NSW 2567, Australia \\ 7 School of Nursing and Midwifery, Western Sydney University, Liverpool Campus, Locked Bag 1797, Penrith, \\ NSW 2571, Australia \\ 8 St John of God Raphael Services, Blacktown, NSW 2148, Australia \\ 9 Ingham Institute for Applied Medical Research, 1 Campbell Street, Liverpool, NSW 2170, Australia \\ 10 School of Women's and Children's Health, The University of New South Wales, Kensington, Sydney, \\ NSW 2052, Australia \\ 11 Menzies Centre for Health Policy, Charles Perkins Centre, School of Public Health, Sydney University, \\ Sydney, NSW 2006, Australia \\ 12 School of Public Health, Griffith University, Gold Coast, QLD 4222, Australia \\ 13 Sydney Institute for Women, Children and their Families, Sydney Local Health District, Camperdown, \\ NSW 2050, Australia \\ * Correspondence: f.ogbo@westernsyndey.edu.au
}

Received: 23 May 2019; Accepted: 11 July 2019; Published: 16 July 2019

\begin{abstract}
There are limited epidemiological data on exclusive breastfeeding (EBF) among culturally and linguistically diverse (CALD) Australian mothers to advocate for targeted and/or culturally-appropriate interventions. This study investigated the determinants of EBF cessation in the early postnatal period among CALD Australian mothers in Sydney, Australia. The study used linked maternal and child health data from two local health districts in Australia $(N=25,407)$. Prevalence of maternal breastfeeding intention, skin-to-skin contact, EBF at birth, discharge, and the early postnatal period (1-4 weeks postnatal), were estimated. Multivariate logistic regression models were used to investigate determinants of EBF cessation in the early postnatal period. Most CALD Australian mothers had the intention to breastfeed (94.7\%). Skin-to-skin contact (81.0\%), EBF at delivery $(91.0 \%)$, and at discharge $(93.0 \%)$ were high. EBF remained high in the early postnatal period $(91.4 \%)$. A lack of prenatal breastfeeding intention was the strongest determinant of EBF cessation (adjusted odds ratio $[\mathrm{aOR}]=23.76,95 \% \mathrm{CI}$ : 18.63-30.30, for mothers with no prenatal breastfeeding intention and $\mathrm{aOR}=6.15,95 \% \mathrm{CI}: 4.74-7.98$, for those undecided). Other significant determinants of EBF cessation included a lack of partner support, antenatal and postnatal depression, intimate partner
\end{abstract}


violence, low socioeconomic status, caesarean birth, and young maternal age ( $<20$ years). Efforts to improve breastfeeding among women of CALD backgrounds in Australia should focus on women with vulnerabilities to maximise the benefits of EBF.

Keywords: exclusive breastfeeding; Australia; skin-to-skin; culturally and linguistically diverse (CALD)

\section{Introduction}

Global health organisations (such as the World Health Organization and the United Nations Children's Fund, WHO/UNICEF) recommend exclusive breastfeeding (EBF) for the first six months of life [1]. EBF is defined as providing the infant human breastmilk only, and when needed, oral rehydration solution, or drops/syrups of vitamins, minerals, or medicines [2]. Breast milk has a high proportion of fat, protein, sugar, and water that is required for infant growth and development compared to formula milk, as well as the immunologic substances to effectively protect against infectious diseases for the infant [3-5]. Appropriate EBF protects against childhood obesity [6] and has the potential to increase infant cognitive functioning [7]. EBF not only benefits the infant but is also associated with improved maternal health (e.g., a reduced risk developing of type 2 diabetes mellitus) [8] and improved household productivity due to no cost of human milk [9].

In Australia, culturally and linguistically diverse (CALD) is a term used for communities with diverse ethnic backgrounds, traditions, food, nationality, language, dress, societal structures, art, and religious characteristics [10]. While it may be debatable that such a 'label' exists to characterize such diverse populations, it is nevertheless one measure that can identify a sub-group in the Australian population who are often socioeconomically vulnerable, and for the purpose of focused research and programmatic interventions [11,12]. Past research suggests that CALD subgroups are more likely to experience adverse health outcomes, including depressive symptoms [13] and poor uptake of cancer screening [14], compared to the non-CALD populations.

Many qualitative studies conducted in Australia have reported that most CALD women value optimal breastfeeding practices, but a lack of access to traditional post-birth practices [15], stigma, and shame around public breastfeeding and ambivalence towards breastfeeding support [16,17], were barriers to appropriate breastfeeding. However, there is limited epidemiological data on the determinants of inappropriate EBF among CALD subgroups to inform targeted interventions. Quantitative research in the general Australian population suggests that intimate partner violence [18], a lack of partner support [18], assisted delivery [18,19], low socioeconomic status [20], lower maternal age (<25 years) [19], a mother not having intention to breastfeeding [21-23], and self-reported depressive symptoms [21] were associated with cessation of EBF. Notably, it is unclear whether these factors are relevant to mothers from CALD backgrounds as culturally-appropriate and focused intervention strategies are potentially more effective and less costly than traditional interventional approaches [12].

Understanding the factors that influence a mother's decision to initiate, cease, or continue EBF in the early postnatal period is essential as it can provide important information and opportunities for specific interventions. In addition to providing relevant data on EBF among CALD Australian mothers, this study seeks to provide an evidence base on EBF among CALD mothers for breastfeeding policy advocacy and/or evaluation of relevant social and health services for mothers of CALD backgrounds in Australia. This study aimed to investigate the determinants of EBF cessation in the early postnatal period among CALD Australian mothers in Sydney, New South Wales. 


\section{Materials and Methods}

\subsection{Data Source}

The study was conducted based on retrospective maternal and child health data of all live births in public health facilities in Sydney Local Health District (SLHD) and South Western Sydney Local Health District (SWSLHD) between 2014 and $2016(N=25,407)$. These data were routinely collected as part of standard care provided during pregnancy and the postnatal period. Antenatal information that included socio-demographic characteristics, history of any previous pregnancy, probable depression based on the Edinburgh Postnatal Depression Scale (EPDS), and mothers' breastfeeding intention were collected by qualified midwives at the first prenatal care visit. Birth and postnatal data such as information on skin-to-skin contact, EBF at discharge and postnatally, were also obtained immediately after birth and during postnatal visits by qualified child and family health nurses (CFHN). During the first prenatal visit, women were asked to identify whether they belong to CALD, non-CALD, or Aboriginal or Torres Strait Islander subpopulations, and this information was entered into the database. CALD population was defined based on the Australian Bureau of Statistics description for the subgroup [10]. These maternal child health $(\mathrm{MCH})$ data were stored in the local health district's Information Management \& Technology Division (IM\&TD) database. We obtained the perinatal data from the IM\&TD, which were cleaned and linked using individual identifiers, and coded for analysis.

\subsection{Study Setting}

In Sydney, the SLHD and SWSLHD cover 52\% of the metropolitan area, with an estimated population of 1.6 million people of different cultural backgrounds [24,25]. SLHD is located in the centre and inner west of Sydney, while SWSLHD is located in the south-western region of Sydney. A number of maternal and child health services are provided to all communities across both districts, including the most socioeconomically disadvantaged populations.

\subsection{Outcome Variables}

The main outcome variable was EBF in the early postnatal period (defined as 1-4 weeks post-birth). EBF was measured using the National Health and Medical Research Council (NHMRC) infant feeding guidelines [26], consistent with the WHO/UNICEF definitions for assessing infant and young child feeding practices [1]. EBF was defined as the proportion of infants who received only breast milk (including expressed milk) but allowed oral rehydration solution, syrups of vitamins/medicines. The prevalence of mothers' breastfeeding intention, skin-to-skin contact, EBF at delivery and discharge by the determining factors were also measured in the study.

EBF at delivery was defined as the proportion of infants who received only breast milk in the first $24 \mathrm{~h}$ post-birth, while EBF at discharge was measured as the proportion of infants who received only breast milk $24 \mathrm{~h}$ preceding discharge from the maternity unit. EBF in the early postnatal visit (1-4 weeks postnatal) referred to the proportion of infants who received only breast milk $24 \mathrm{~h}$ prior to this postnatal health visit by the CFHN. To assess mothers' breastfeeding intention, they were asked the following question: "Do you plan to breastfeed your child?" The study also considered skin-to-skin contact as past studies have indicated that skin-to-skin contact is the most effective strategy to promote, protect, and support EBF in early life, irrespective of the mode of birthing (vaginal or caesarean births) [27-29]. Skin-to-skin contact (SSC) was defined as placing the naked baby on the mother's bare chest or abdomen immediately or less than $10 \mathrm{~min}$ after birth or soon afterwards [30].

In each local health district, assessment of both the mother and baby is conducted between the first and fourth week post-birth by a CFHN during a universal health home visit. Relevant health information (including anthropometric measurement of the baby and assessment of infant feeding practices of the mother) are obtained and entered into the IM\&TD database. 


\subsection{Study Factors}

The exposure variables were broadly categorised into socio-demographic and health factors, which were selected based on previous studies $[18,19,22,23]$ and data availability. Socio-demographic factors included maternal age (categorised as $<20$ years, 20-34 years or $\geq 35$ years), socioeconomic status (SES, categorised as high, middle or low), maternal cigarette smoking in pregnancy (categorised as yes or no), partner support (categorised as yes, not sure or no), and major nationality groups (categorised as Oceania, North-West Europe, Southern-Eastern Europe, North Africa and The Middle East, South-East Asia, North-East Asia, Southern and Central Asia, Americas or Sub-Saharan Africa).

Health factors included pre-existing maternal health problems (such as diabetes mellitus and/or hypertension, categorised as yes or no), history of intimate partner violence (IPV, categorised as yes or no), type of delivery (categorised as normal vaginal, assisted vaginal or caesarean), and self-reported antenatal and postnatal depressive symptoms (categorised as score $\geq 13$ or score $<13$ on the EPDS) [31]. Maternal breastfeeding intention was also considered a potential factor for the cessation of EBF in the early post-birth period based on past studies [21-23].

SES was calculated using the Socio-Economic Index for Areas (SEIFA). SEIFA is an indicator created by the Australian Bureau of Statistics using principal component analysis. It ranks areas (including a mother's address provided) in Australia according to relative socio-economic advantage and disadvantage [32]. In this study, deciles of SES were categorised into high (top $10 \%$ of the population), middle (middle $80 \%$ ), and low (bottom 10\%) groups, in line with previously published studies [13,31]. In accordance with NSW Health policy [33], IPV information was collected from mothers based on the following questions: (i) "within the last year have you been hit, slapped or hurt in other ways by your partner or ex-partner?", physical IPV; and (ii) "are you frightened of your partner or ex-partner?", psychological IPV.

\subsection{Statistical Analysis}

The analytical approach followed previously published studies [18,31]. Briefly, preliminary analyses were conducted to calculate frequencies of the study outcomes (i.e., breastfeeding intention, skin-to-skin contact, and EBF at delivery, discharge and postnatally) and cross-tabulations with study factors. This was followed by univariate regression models to examine the association between each study factor and cessation of EBF in the early postnatal period. Multivariate logistic regression analyses that adjusted for confounders was conducted to investigate the potential study factors that were associated with cessation of EBF in the early postnatal period among CALD mothers. Models adjusted for the potential confounding factors of birthing facility, the gender of the baby, maternal alcohol intake, and maternal body mass index, as well as socio-economic and health factors [21,34]. Odds ratios, with $95 \%$ confidence intervals, were calculated as the measure of association between the risk factors and cessation of EBF.

Our study also investigated the potential effect of missing data on the estimated odds ratios in sensitivity analyses that used an imputed dataset, based on the original data, which comprised complete information for EBF in the early postnatal period. Multiple imputations by chained equations were employed, which assume that data were missing at random [35]. This analytical approach also assumes that the known characteristics of study respondents can be used to examine the characteristics of participants with missing data [36]. All study factors and the outcome variable in the main analysis were included in the multiple imputation models. Revised odds ratios from the imputed data were generated using the mim command, for comparison with the complete case analyses. Sensitivity analyses were conducted based on 25 multiple imputations [37], and all analyses were conducted in Stata (Stata Corp, version 15.0, College Station, TX, USA). 


\subsection{Ethics}

The Sydney Local Health District and South Western Sydney Local Health District Human Research Ethics Committees approved the collection of the data from the IM\&TD database and subsequent analysis. Approval numbers HREC: LNR/11/LPOOL/463; SSA: LNRSSA/11/LPOOL/464 and Project No: 11/276 LNR; Protocol No X12-0164 and LNR/12/RPAH/266.

\section{Results}

\subsection{Characteristics of the Study Population}

The majority of mothers were from South-East Asia (24.5\%) and North Africa and The Middle East $(23.0 \%)$, while the lowest proportion of mothers were from North-West Europe (1.5\%) (Table 1).

Table 1. Characteristics of the study population $(N=25,407)$.

\begin{tabular}{|c|c|c|}
\hline Variables & $\mathbf{N}$ & $(\%)$ \\
\hline \multicolumn{3}{|l|}{ Sociodemographic factors } \\
\hline Maternal age group & 25,407 & \\
\hline 20-34 years & 23,812 & 93.7 \\
\hline$<20$ years & 124 & 0.5 \\
\hline$\geq 35$ years & 1471 & 5.8 \\
\hline SES category & 23,786 & \\
\hline Low & 12,548 & 52.8 \\
\hline Middle & 9377 & 39.4 \\
\hline High & 1861 & 7.8 \\
\hline Smoking status & 23,750 & \\
\hline No & 23,170 & 97.6 \\
\hline Yes & 580 & 2.4 \\
\hline Supportive partner & 21,672 & \\
\hline Yes & 21,061 & 97.2 \\
\hline No & 611 & 2.8 \\
\hline Major nationality group & 25,407 & \\
\hline Oceania & 1481 & 5.8 \\
\hline North-West Europe & 384 & 1.5 \\
\hline Southern-Eastern Europe & 1316 & 5.2 \\
\hline North Africa and The Middle East & 5846 & 23.0 \\
\hline South-East Asia & 6222 & 24.5 \\
\hline North-East Asia & 3512 & 13.8 \\
\hline Southern and Central Asia & 5127 & 20.2 \\
\hline Americas & 634 & 2.5 \\
\hline Sub-Saharan Africa & 885 & 3.5 \\
\hline \multicolumn{3}{|l|}{ Health factors } \\
\hline Antenatal health problems & 24,603 & \\
\hline No & 20,517 & 83.4 \\
\hline Yes & 4086 & 16.6 \\
\hline Psychosocial intimate partner violence & 21,583 & \\
\hline No & 21,238 & 98.4 \\
\hline Yes & 345 & 1.6 \\
\hline Physical intimate partner violence & 21,523 & \\
\hline No & 21,240 & 98.7 \\
\hline Yes & 283 & 1.3 \\
\hline Type of delivery & 25,378 & \\
\hline Normal vaginal & 15,004 & 59.1 \\
\hline Assisted vaginal & 2924 & 11.5 \\
\hline Caesarean section & 7450 & 29.4 \\
\hline
\end{tabular}


Table 1. Cont

\begin{tabular}{lll}
\hline \multicolumn{1}{c}{ Variables } & \multicolumn{1}{c}{$\mathbf{N}$} & \multicolumn{1}{c}{$(\mathbf{\%})$} \\
\hline Antenatal depressive symptoms & 20,560 & \\
\hline EPDS $\leq 9$ & 16,972 & 82.6 \\
\hline EPDS $10-12$ & 2078 & 10.1 \\
\hline EPDS $\geq 13$ & 1510 & 7.3 \\
\hline Postnatal depressive symptoms & 19,342 & \\
\hline EPDS $\leq 9$ & 17,425 & 90.1 \\
\hline EPDS $10-12$ & 1194 & 6.2 \\
\hline EPDS $\geq 13$ & 723 & 3.7 \\
\hline
\end{tabular}

$\mathrm{N}$ = Sample size; EPDS: Edinburgh Postnatal Depression Scale; SES: Socioeconomic status.

\subsection{Breastfeeding Patterns by Study Factors}

The proportion of CALD Australian mothers who exclusively breastfed in the early postnatal period varied by global regions, with mothers from South-East Asia (26.9\%), North Africa and the Middle East (33.8\%), and Oceania (12.2\%) representing the highest percentage (Table 2). Mothers from the high SES category had a higher proportion of EBF (63.1\%) compared to those from middle and low SES categories (33.4\% and 3.5\%, respectively).

Table 2. Prevalence of breastfeeding among culturally and linguistically diverse (CALD) mothers from South Western Sydney and Sydney Local Health Districts in Sydney, Australia, 2014-2016 $(N=25,407)$.

\begin{tabular}{|c|c|c|c|c|c|}
\hline Variables & $\begin{array}{l}\text { Breastfeeding } \\
\text { Intention }\end{array}$ & $\begin{array}{l}\text { Skin-to-Skin } \\
\text { Contact }\end{array}$ & $\begin{array}{c}\text { EBF at } \\
\text { Delivery }\end{array}$ & $\begin{array}{c}\text { EBF at } \\
\text { Discharge }\end{array}$ & $\begin{array}{l}\text { EBF at 1-4 } \\
\text { Weeks }\end{array}$ \\
\hline & n (\%) & n $(\%)$ & n (\%) & n (\%) & n (\%) \\
\hline \multicolumn{6}{|l|}{ Study outcomes } \\
\hline Yes & $21,232(94.7)$ & $16,022(81.2)$ & $19,355(90.7)$ & $22,555(93.3)$ & $19,244(91.4)$ \\
\hline No & $629(2.8)$ & $3697(18.8)$ & $1973(9.3)$ & $1629(6.7)$ & $1809(8.6)$ \\
\hline Undecided & $572(2.5)$ & - & - & - & - \\
\hline \multicolumn{6}{|l|}{ Socio-demographic factors } \\
\hline \multicolumn{6}{|l|}{ Maternal age group } \\
\hline 20-34 years & $21,048(93.8)$ & $18,651(94.6)$ & $20,023(93.9)$ & $22,690(93.8)$ & $1628(90.0)$ \\
\hline$<20$ years & $103(0.5)$ & $113(0.6)$ & $112(0.5)$ & $1375(5.7)$ & $14(9.2)$ \\
\hline$\geq 35$ years & $1282(5.7)$ & $955(4.8)$ & $1193(5.6)$ & $119(0.5)$ & $167(0.8)$ \\
\hline \multicolumn{6}{|l|}{ SES category } \\
\hline High & $10,304(63.4)$ & $9778(52.9)$ & $10,411(52.1)$ & $11,877(52.5)$ & $1109(63.1)$ \\
\hline Middle & $7962(32.8)$ & 7268 (39.3) & $7958(39.8)$ & $8922(39.5)$ & $588(33.4)$ \\
\hline Low & $1598(3.8)$ & $1427(7.7)$ & $1612(8.1)$ & $1817(8.0)$ & $62(3.5)$ \\
\hline \multicolumn{6}{|l|}{ Smoking status } \\
\hline No & $21,860(97.6)$ & $17,937(97.3)$ & $19,520(97.7)$ & $22,207(97.6)$ & $1532(92.9)$ \\
\hline Yes & $543(2.4)$ & $437(2.4)$ & $467(2.3)$ & $540(2.4)$ & $117(7.1)$ \\
\hline \multicolumn{6}{|l|}{ Supportive partner } \\
\hline Yes & $20,501(97.2)$ & $16,457(97.2)$ & $17,773(97.2)$ & $20,190(97.3)$ & $1454(95.7)$ \\
\hline No & $585(2.8)$ & $481(2.8)$ & $508(2.8)$ & $569(2.7)$ & $66(4.3)$ \\
\hline \multicolumn{6}{|l|}{ Major nationality group } \\
\hline Oceania & $1237(5.5)$ & $1193(6.1)$ & $1247(5.9)$ & $1368(5.7)$ & $221(12.2)$ \\
\hline North-West Europe & $360(1.6)$ & $326(1.7)$ & $343(1.6)$ & $373(1.5)$ & $18(1.0)$ \\
\hline Southern-Eastern Europe & $1177(5.3)$ & $1028(5.2)$ & $1146(5.4)$ & $1262(5.2)$ & $128(7.08)$ \\
\hline North Africa and The Middle East & $5100(22.7)$ & $4649(23.6)$ & $4901(23.0)$ & $5526(22.8)$ & $612(33.8)$ \\
\hline South-East Asia & $5437(24.2)$ & $4958(25.1)$ & $5235(24.6)$ & $5868(24.3)$ & $487(26.9)$ \\
\hline North-East Asia & $3150(14.0)$ & $2837(14.4)$ & $3048(14.3)$ & $3431(14.2)$ & $150(8.3)$ \\
\hline
\end{tabular}


Table 2. Cont.

\begin{tabular}{|c|c|c|c|c|c|}
\hline Variables & $\begin{array}{l}\text { Breastfeeding } \\
\text { Intention }\end{array}$ & $\begin{array}{l}\text { Skin-to-Skin } \\
\text { Contact }\end{array}$ & $\begin{array}{c}\text { EBF at } \\
\text { Delivery }\end{array}$ & $\begin{array}{c}\text { EBF at } \\
\text { Discharge }\end{array}$ & $\begin{array}{l}\text { EBF at 1-4 } \\
\text { Weeks }\end{array}$ \\
\hline & n (\%) & n (\%) & n (\%) & n (\%) & n (\%) \\
\hline Southern and Central Asia & $4636(20.7)$ & 3638 (18.5) & 4158 (19.5) & $4914(20.3)$ & $116(6.4)$ \\
\hline Americas & $552(2.5)$ & $439(2.2)$ & $526(2.5)$ & $607(2.5)$ & $42(2.3)$ \\
\hline Sub-Saharan Africa & $784(3.5)$ & $651(3.3)$ & $724(3.4)$ & $835(3.5)$ & $35(1.9)$ \\
\hline \multicolumn{6}{|l|}{ Health factors } \\
\hline \multicolumn{6}{|l|}{ Antenatal health problems } \\
\hline No & $17,847(82.4)$ & $15,821(83.0)$ & $17,334(83.7)$ & $19,514(83.3)$ & $1496(85.1)$ \\
\hline Yes & 3819 (17.6) & $3245(17.0)$ & $3370(16.3)$ & $3916(16.7)$ & $263(14.9)$ \\
\hline \multicolumn{6}{|c|}{$\begin{array}{l}\text { Psychosocial intimate partner } \\
\text { violence }\end{array}$} \\
\hline No & $20,657(98.4)$ & $19,618(98.4)$ & $17,919(98.4)$ & $20,360(98.5)$ & $1517(97.9)$ \\
\hline Yes & 335 (1.6) & $273(1.6)$ & $290(1.6)$ & $316(1.5)$ & $33(2.1)$ \\
\hline \multicolumn{6}{|c|}{ Physical intimate partner violence } \\
\hline No & $20,663(98.7)$ & $16,616(98.7)$ & $17,911(98.7)$ & $20,356(98.7)$ & $1522(98.1)$ \\
\hline Yes & $270(1.3)$ & $213(1.3)$ & $243(1.3)$ & $265(1.3)$ & $30(1.9)$ \\
\hline \multicolumn{6}{|l|}{ Type of delivery } \\
\hline Normal vaginal & $13,356(59.6)$ & $14,903(75.7)$ & $14,126(66.3)$ & $14,432(59.7)$ & $988(54.7)$ \\
\hline Assisted vaginal & $2581(11.5)$ & $2878(14.6)$ & $2452(11.5)$ & $2759(11.4)$ & $163(9.0)$ \\
\hline Caesarean section & $6473(28.9)$ & $1913(9.7)$ & $4730(22.2)$ & $6968(28.8)$ & $655(36.3)$ \\
\hline \multicolumn{6}{|c|}{ Antenatal depressive symptoms } \\
\hline $\mathrm{EPDS} \leq 9$ & $16,543(72.6)$ & $13,406(83.3)$ & $14,392(82.9)$ & $16,259(82.6)$ & $1164(79.6)$ \\
\hline EPDS 10-12 & $2013(10.1)$ & $1577(9.8)$ & $1751(10.1)$ & $1992(10.1)$ & $146(10.0)$ \\
\hline EPDS $\geq 13$ & $1465(7.3)$ & $1121(6.9)$ & $1222(7.0)$ & $1433(7.3)$ & $152(10.4)$ \\
\hline \multicolumn{6}{|c|}{ Postnatal depressive symptoms } \\
\hline EPDS $\leq 9$ & $15,451(90.2)$ & $13,538(90.6)$ & $14,739(90.5)$ & $16,628(90.2)$ & $1,489(88.9)$ \\
\hline EPDS 10-12 & $1055(6.2)$ & $880(5.9)$ & $973(6.0)$ & $1139(6.2)$ & $94(5.6)$ \\
\hline$E P D S \geq 13$ & $633(3.7)$ & $523(3.5)$ & $579(3.5)$ & 677 (3.6) & $92(5.5)$ \\
\hline
\end{tabular}

$\mathrm{n}$ : cases; antenatal health problems included diabetes mellitus and/or hypertension; EBF at delivery was defined as infants who received only breast milk within the first $24 \mathrm{~h}$ post-delivery; EBF at discharge was measured as infants who received only breast milk in the $24 \mathrm{~h}$ preceding discharge from the maternity unit; EPDS: Edinburgh Postnatal Depression Scale; SES: Socioeconomic status.

In the antenatal period, almost all mothers intended to breastfeed their babies $(94.7 \%)$. Approximately $81 \%$ of mothers practised skin-to-skin contact, while $91 \%$ and $93 \%$ exclusively breastfed at delivery and discharge, respectively. A sub-analysis of the data (i.e., the estimation of the confidence interval and $p$-value around the estimates, data not shown) suggested that there was no significant difference between EBF prevalence at delivery and discharge. In the early postnatal period, EBF remained high among CALD Australian mothers (91.4\%; Table 2).

\subsection{Determinants of Exclusive Breastfeeding Cessation in the Early Postnatal Period}

The study showed that mothers who indicated no prenatal breastfeeding intention or who were undecided about breastfeeding during pregnancy were more likely to cease EBF in the early postnatal period compared to those who indicated prenatal breastfeeding intention in the complete case analyses (adjusted odds ratio $(\mathrm{aOR})=23.76,95 \% \mathrm{CI} 18.63-30.30, p<0.001$, for those with no prenatal breastfeeding intention and $\mathrm{aOR}=6.15,95 \% \mathrm{CI} 4.74-7.98, p<0.001$, for those undecided) (Table 3). 
Table 3. Determinants of exclusive breastfeeding cessation in the early postnatal period among CALD mothers in South Western Sydney and Sydney Local Health Districts, 2014-2016 ( $N=25,407)$.

\begin{tabular}{|c|c|c|c|c|c|c|c|c|}
\hline \multirow[t]{2}{*}{ Study Factors } & \multicolumn{2}{|c|}{ Complete Case Analysis } & \multicolumn{4}{|c|}{$\begin{array}{c}\text { Multiple } \\
\text { Imputation } \\
\text { Analysis * }\end{array}$} & \multirow[b]{2}{*}{$\begin{array}{c}\text { Adjusted OR } \\
(95 \% \text { CI) (a) }\end{array}$} & \multirow[b]{2}{*}{$p$ Value } \\
\hline & $\begin{array}{l}\text { Unadjusted OR } \\
\quad(95 \% \mathrm{CI})\end{array}$ & $p$ Value & $\begin{array}{c}\text { Adjusted OR } \\
(95 \% \text { CI) (a) }\end{array}$ & $p$ Value & $\begin{array}{l}\text { Unadjusted OR } \\
\quad(95 \% \mathrm{CI})\end{array}$ & $p$ Value & & \\
\hline \multicolumn{9}{|l|}{ Antenatal breastfeeding intention } \\
\hline Yes & 1.00 & & 1.00 & & 1.00 & & 1.00 & \\
\hline No & $31.11(25.41-38.10)$ & $<0.001$ & $23.76(18.63-30.30)$ & $<0.001$ & $31.11(29.90-32.37)$ & $<0.001$ & $27.13(26.04-28.27)$ & $<0.001$ \\
\hline Undecided & $7.01(5.69-8.64)$ & $<0.001$ & $6.15(4.74-7.98)$ & $<0.001$ & $7.00(6.72-7.30)$ & $<0.001$ & $6.38(6.11-6.66)$ & $<0.001$ \\
\hline Yes & 1.00 & & 1.00 & & 1.00 & & 1.00 & \\
\hline No & $1.81(1.38-2.36)$ & $<0.001$ & $1.69(1.20-2.38)$ & 0.003 & $1.66(1.58-1.74)$ & $<0.001$ & $1.60(1.52-1.69)$ & $<0.001$ \\
\hline \multicolumn{9}{|l|}{ Socio-economic status } \\
\hline Low & 1.00 & & 1.00 & & & & & \\
\hline Middle & $0.67(0.61-0.75)$ & $<0.001$ & $0.85(0.72-0.99)$ & 0.044 & $0.67(0.65-0.68)$ & $<0.001$ & $0.84(0.82-0.86)$ & $<0.001$ \\
\hline High & $0.36(0.28-0.47)$ & $<0.001$ & $0.48(0.32-0.71)$ & $<0.001$ & $0.36(0.34-0.38)$ & $<0.001$ & $0.59(0.56-0.63)$ & $<0.001$ \\
\hline \multicolumn{9}{|l|}{ Maternal age group } \\
\hline \multicolumn{9}{|l|}{ Major nationality group } \\
\hline Oceania & 1.00 & & & & 1.00 & & & \\
\hline North-West Europe & $0.25(0.15-0.41)$ & $<0.001$ & $0.49(0.25-0.97)$ & 0.043 & $0.25(0.23-0.28)$ & $<0.001$ & $0.47(0.43-0.52)$ & $<0.001$ \\
\hline Southern-Eastern Europe & $0.57(0.45-0.73)$ & $<0.001$ & $0.73(0.53-1.00)$ & 0.051 & $0.57(0.55-0.60)$ & $<0.001$ & $0.71(0.68-0.74)$ & $<0.001$ \\
\hline North Africa and The Middle East & $0.67(0.56-0.79)$ & $<0.001$ & $0.65(0.51-0.82)$ & $<0.001$ & $0.67(0.65-0.69)$ & $<0.001$ & $0.64(0.62-0.67)$ & $<0.001$ \\
\hline South-East Asia & $0.45(0.38-0.53)$ & $<0.001$ & $0.46(0.36-0.58)$ & $<0.001$ & $0.45(0.43-0.46)$ & $<0.001$ & $0.47(0.45-0.49)$ & $<0.001$ \\
\hline North-East Asia & $0.24(0.19-0.31)$ & $<0.001$ & $0.41(0.29-0.56)$ & $<0.001$ & $0.24(0.23-0.26)$ & $<0.001$ & $0.43(0.41-0.45)$ & $<0.001$ \\
\hline Southern and Central Asia & $0.11(0.09-0.14)$ & $<0.001$ & $0.18(0.13-0.24)$ & $<0.001$ & $0.11(0.11-0.12)$ & $<0.001$ & $0.14(0.14-0.15)$ & $<0.001$ \\
\hline Americas & $0.37(0.26-0.53)$ & $<0.001$ & $0.40(0.24-0.66)$ & $<0.001$ & $0.37(0.35-0.40)$ & $<0.001$ & $0.41(0.38-0.44)$ & $<0.001$ \\
\hline Sub-Saharan Africa & $0.21(0.14-0.31)$ & $<0.001$ & $0.25(0.15-0.41)$ & $<0.001$ & $0.21(0.20-0.23)$ & $<0.001$ & $0.24(0.23-0.26)$ & $<0.001$ \\
\hline \multicolumn{9}{|l|}{ Health factors } \\
\hline \multicolumn{9}{|l|}{ Antenatal depressive symptoms } \\
\hline EPDS $\leq 9$ & 1.00 & & 1.00 & & 1.00 & & 1.00 & \\
\hline EPDS 10-12 & $1.03(0.86-1.23)$ & 0.783 & $1.10(0.88-1.36)$ & 0.398 & $1.02(0.99-1.06)$ & 0.161 & $1.00(0.97-1.04)$ & $<0.001$ \\
\hline
\end{tabular}


Table 3. Cont

\begin{tabular}{|c|c|c|c|c|c|c|c|c|}
\hline \multirow[t]{2}{*}{ Study Factors } & \multicolumn{2}{|c|}{ Complete Case Analysis } & \multicolumn{4}{|c|}{$\begin{array}{l}\text { Multiple } \\
\text { Imputation } \\
\text { Analysis* }\end{array}$} & \multirow[b]{2}{*}{$\begin{array}{c}\text { Adjusted OR } \\
(95 \% \text { CI) (a) }\end{array}$} & \multirow[b]{2}{*}{$p$ Value } \\
\hline & $\begin{array}{l}\text { Unadjusted OR } \\
(95 \% \mathrm{CI})\end{array}$ & $p$ Value & $\begin{array}{l}\text { Adjusted OR } \\
\text { (95\% CI) (a) }\end{array}$ & $p$ Value & $\begin{array}{l}\text { Unadjusted OR } \\
(95 \% \mathrm{CI})\end{array}$ & $p$ Value & & \\
\hline \multicolumn{9}{|c|}{ Postnatal depressive symptoms } \\
\hline EPDS $\leq 9$ & 1.00 & & 1.00 & & 1.00 & & 1.00 & \\
\hline EPDS 10-12 & $0.911(0.73-1.13)$ & 0.402 & $0.97(0.73-1.30)$ & 0.860 & $0.92(0.88-0.96)$ & $<0.001$ & $1.00(0.96-1.04)$ & 0.885 \\
\hline EPDS $\geq 13$ & $1.61(1.28-2.01)$ & $<0.001$ & $2.07(1.55-2.77)$ & $<0.001$ & $1.57(1.50-1.63)$ & $<0.001$ & $1.66(1.59-1.73)$ & $<0.001$ \\
\hline \multicolumn{9}{|c|}{$\begin{array}{l}\text { Psychosocial intimate partner } \\
\text { violence }\end{array}$} \\
\hline No & 1.00 & & 1.00 & & 1.00 & & 1.00 & \\
\hline Yes & $1.47(1.02-2.12)$ & 0.041 & $1.66(1.10-2.53)$ & 0.017 & $1.46(1.36-1.57)$ & $<0.001$ & $1.50(1.39-1.61)$ & $<0.001$ \\
\hline \multicolumn{9}{|c|}{ Physical intimate partner violence } \\
\hline No & 1.00 & & 1.00 & & 1.00 & & 1.00 & \\
\hline Yes & $1.57(1.06-2.31)$ & 0.023 & $1.49(0.94-2.38)$ & 0.093 & $1.56(1.45-1.69)$ & $<0.001$ & $1.62(1.50-1.75)$ & $<0.001$ \\
\hline \multicolumn{9}{|l|}{ Type of delivery } \\
\hline Normal vaginal & 1.00 & & 1.00 & & 1.00 & & 1.00 & \\
\hline Assisted vaginal & $0.79(0.67-0.94)$ & 0.008 & $0.90(0.72-1.14)$ & 0.386 & $0.79(0.76-0.82)$ & $<0.001$ & $0.96(0.88-0.92)$ & 0.078 \\
\hline Caesarean section & $1.36(1.23-1.51)$ & $<0.001$ & $1.35(1.18-1.55)$ & $<0.001$ & $1.36(1.33-1.39)$ & $<0.001$ & $1.48(1.45-1.52)$ & $<0.001$ \\
\hline \multicolumn{9}{|c|}{$\begin{array}{l}\text { Pre-existing maternal health } \\
\text { problems }\end{array}$} \\
\hline No & 1.00 & & 1.00 & & 1.00 & & 1.00 & \\
\hline Yes & $0.87(0.76-1.00)$ & 0.054 & $1.02(0.83-1.07)$ & 0.790 & $0.87(0.85-0.89)$ & $<0.001$ & $0.95(0.93-0.98)$ & 0.004 \\
\hline
\end{tabular}

(a): adjusted for maternal body mass index, gender of the baby, maternal alcohol intake, Aboriginality and birthing facility location, as well as socioeconomic and health factors. * Sensitivity analyses following multiple imputations for missing values; pre-existing maternal health problems included diabetes mellitus and/or hypertension. 
Mothers who reported not having a supportive partner during pregnancy were more likely to stop EBF in the early post-birth period compared to those who reported receiving support from their partner during pregnancy ( $\mathrm{aOR}=1.69,95 \% \mathrm{CI}: 1.20-2.38, p=0.003)$. Mothers from higher SES groups were less likely to discontinue EBF in the early postnatal period compared to those from lower SES groups (aOR $=0.48,95 \%$ CI: $0.32-0.71, p<0.001$ for high SES and $\mathrm{aOR}=0.85,95 \%$ CI: $0.85-0.99$, $p=0.044$ for middle). Low maternal age ( $<20$ years) was associated with cessation of EBF in the early postnatal period compared to middle reproductive-aged mothers (20-34 years; aOR $=1.72,95 \% \mathrm{CI}$ : $1.37-2.15, p<0.001)$. Mothers who reported tobacco smoking during pregnancy were more likely to stop EBF in the early postnatal period compared to their counterparts who reported not smoking $(\mathrm{aOR}=3.39,95 \%$ CI: $2.56-4.49, p<0.001)$ (Table 3$)$.

The odds of stopping EBF in the immediate postpartum period were higher among mothers who reported antenatal depressive symptoms (EPDS $\geq 13$; aOR $=1.50,95 \%$ CI: $1.20-1.89, p<0.001$ ), and those who reported postnatal depressive symptoms (EPDS $\geq 13$; aOR $=2.07,95 \%$ CI: 1.55-2.77, $p<0.001)$. The likelihood of discontinuing EBF in the immediate postnatal period was higher among mothers who reported a history of psychological intimate partner violence (aOR $=1.66,95 \% \mathrm{CI}$ : $1.10-2.53, p=0.017$ ), and those who had caesarean births (aOR $=1.35,95 \%$ CI: $1.18-1.55, p<0.001$ ). The odds of ceasing EBF in the immediate postpartum period were lower among mothers from all major nationality groups compared to their counterparts in the reference group (Oceania) (Table 3). The effects of maternal age $>35$ years, physical intimate partner violence, and having pre-existing maternal health problems on ceasing EBF in the immediate postnatal period were statistically significant in the imputation data compared to those in the complete case analysis. Furthermore, the $95 \% \mathrm{CI}$ of the aOR for no maternal breastfeeding intention and major nationality group based on the imputation data is much narrower than the one obtained based on the original data set, possibly reflecting the effect of missing data or small sample size (Table 3).

\section{Discussion}

Our study indicates that most CALD Australian mothers had the intention to breastfeed (94.7\%). Eighty-one percent of mothers practised skin-to-skin contact, while $91 \%$ and $93 \%$ exclusively breastfed at delivery and discharge, respectively. Notably, EBF remained high in the early postnatal period among CALD Australian mothers (91.4\%). A lack of maternal prenatal breastfeeding intention (no prenatal breastfeeding intention or undecided) was the strongest risk factor for the cessation of EBF in the early postpartum period. Other significant factors associated with the cessation of EBF in the early postnatal period included a lack of partner support, antenatal and postnatal depressive symptoms, psychosocial IPV, caesarean birthing, low socioeconomic status, and young maternal age ( $<20$ years).

Consistent with past reports from Australia [21] and internationally [38-40], the present study indicates that a mother's prenatal intention not to breastfeed or undecided were the strongest risk factors for the cessation of EBF in the early postnatal period among CALD Australian women. Studies suggest that a good personal attitude towards breastfeeding, encouraging social norms for breastfeeding at home and work and a personal dislike for formula feeding, were essential to a mother's decision to have a definite intention to breastfeed [38,41]. In addition, the level of social support for the mother at the time of breastfeeding from the partner, grandmothers/in-laws [42], or peers [43,44], and the mother's own attitude to breastfeeding were influential to a mother's breastfeeding behaviour [38]. Our study also suggests that the lack of a supportive partner was associated with cessation of EBF in the early postnatal period. While studies have indicated that partners are willing to provide the required support to improve breastfeeding outcomes for both the mother and baby, a lack of appropriate and/or conflicting information from health practitioners to fathers have been flagged as constraints to fathers' full participation in breastfeeding support $[45,46]$. The involvement of fathers (in addition to grandmothers/in-laws if present) in prenatal breastfeeding education sessions and postnatal support are key priority areas for improving breastfeeding among mothers [47]. 
In Australia, many epidemiological studies have indicated that higher maternal SES was associated with EBF $[19,20]$. Our study shows that CALD mothers from higher SES groups were less likely to cease EBF in the early postnatal period compared to those from lower SES groups. Similarly, evidence has shown that infants whose fathers were from high SES groups were more likely to be breastfed up to 6-weeks post-birth compared to infants with fathers of low SES [47]. Possible reasons for why higher SES mothers (CALD and non-CALD) engage in optimal breastfeeding may include increased uptake of breastfeeding-related information and better skills in negotiating flexible workplace hours, creating opportunities for breastfeeding [48,49]. Research shows that young maternal age ( $<20$ years) was associated with increased risk of mental health issues, with subsequent impacts on employment and socioeconomic position in later life [50]. The present study shows that young maternal age ( $<20$ years) is associated with cessation of EBF in the early postnatal period, in line with previously published studies $[18,19]$. In many Australian communities, charitable organisations provide parenting support, life support, and educational opportunities for young mothers and their babies to lead healthy and productive lives in their local communities [51]. Integration of these services with antenatal and postnatal breastfeeding services (where practicable) would be essential to improve breastfeeding outcomes among young mothers.

Our study demonstrates an association between self-reported maternal antenatal and postnatal depressive symptoms (EPDS $\geq 13$ ) and cessation of EBF within 4-weeks postnatally. Past studies have indicated that perinatal depression is associated with suboptimal breastfeeding, including cessation of EBF in the early postnatal period [52-54]. Our finding highlights the importance of routine perinatal depression screening in Australia [55]. Detailed information on policy and research implications of Australian perinatal depression screening has been published elsewhere $[13,31,55,56]$. Furthermore, the present study shows that self-reported psychological intimate partner violence is associated with cessation of EBF in the early postnatal period, in line with previous studies $[33,57,58]$. In most Australian healthcare centres, routine screening of women in the antepartum and postpartum periods for depression $[13,55]$ and IPV $[33,59]$ is conducted in an effort to promptly identify at-risk mothers, ensure referral for clinical assessment, follow-up, and support. Our findings suggest that sustained advocacy is required for perinatal depression and IPV screening among Australian CALD women to improve EBF.

Past studies conducted in Australia and internationally, have shown that cigarette smoking is associated with suboptimal breastfeeding outcomes $[18,60]$. Similarly, the present study shows that cigarette smoking is associated with cessation of EBF in the early postnatal period among CALD mothers. Evidence suggests that while there is an association between cigarette smoking and suboptimal breastfeeding, the evidence for a plausible biological mechanism is weak [61]. Nevertheless, a recent review indicated that smoking reduces the protective effects of breast milk and also negatively modifies the infant's response to breastfeeding and breast milk [62]. A possible explanation for this association among smokers may stem from a lack of motivation to breastfeed (i.e., less likely to have breastfeeding intention or to initiate breastfeeding) and/or less desire to seek help with breastfeeding challenges compared to non-smokers $[60,61,63]$. Australia has legislated a range of anti-smoking strategies (including restrictions on advertising, incremental taxation and regulation) to reduce smoking in the community. These initiatives have been shown to be effective in reducing experimentation and uptake of smoking among young people and overall smoking rates across all socio-demographics in Australia [63]. Sustained implementation of these initiatives will have positive impacts on breastfeeding outcomes among CALD Australian mothers in the short- and long-term.

There is robust evidence in the literature which indicates that health facility birthing, mode of birthing, and professional assistance received during birthing are critical to a mother's breastfeeding initiation and continuation $[18,64]$. This possibly reflects the key role the Baby Friendly Hospital Initiative (BFHI) plays in promoting, protecting, and supporting optimal breastfeeding in health facilities. Our study found that caesarean birthing was associated with cessation of EBF in the early postpartum period, which is similar to findings from previous studies [18,19]. Among Australian states 
and territories, evidence indicates that NSW has the lowest number of BFHI certified maternity centres, with implications for maternal breastfeeding experiences [18]. It is anticipated that the Australian National Breastfeeding Strategy 2019 and beyond will provide a roadmap for improving breastfeeding outcomes among CALD mothers in Australia, including increasing BFHI certified maternity centres in NSW [65]. The study showed that the odds of stopping EBF in the immediate postpartum period were lower among mothers from all major nationality groups compared to their counterparts in the reference group (Oceania). Future studies that focus on breastfeeding outcomes among sub-groups within the CALD populations may be warranted, as they would provide more insights for cultural care.

Limitations of this study are discussed. First, the cessation of breastfeeding in the early postnatal period was based on self-report, potentially leading a recall and/or measurement bias. The implication of this is that it may have resulted in an underestimation or overestimation of the relationship between the risk factors and the outcome. Second, the study was unable to assess or adjust for all potential determining factors (e.g., prematurity, level of support received postnatally, multi-parity, or partner education) as these may also affect the observed results. Third, we were unable to distinguish mothers who ceased EBF in the first, second, third, or fourth week postpartum. This analysis would have provided detailed information on early cessation of EBF postnatally among CALD Australian mothers. Fourth, the small sample size of mothers who indicated no prenatal breastfeeding intention or who were undecided may account for the large effect size. Fifth, the non-use of maternal and child health data from private healthcare and other local health districts in Sydney is a limitation in the present study. Finally, the unavailability of longitudinal data on EBF from 1 to 6 months postnatally and the use of secondary data were additional limitations in this study. Despite these limitations, the study provides breastfeeding data from CALD Australian women to inform population-level interventional strategies.

\section{Conclusions}

Our study suggests that many CALD mothers have definite intention to breastfeed, and most mothers practise skin-to-skin contact, EBF at delivery and at discharge from hospital post-birth. Notably, EBF remained high in the early postnatal period (within 4-weeks postpartum) among CALD Australian mothers. A lack of maternal prenatal breastfeeding intention and partner support, antenatal and postnatal depressive symptoms, psychosocial intimate partner violence, caesarean birthing, low socioeconomic status, and young maternal age ( $<20$ years) were associated with the cessation of EBF in the early postnatal period among CALD Australian mothers. Our study provides insight into breastfeeding practices among CALD Australian mothers to inform targeted initiatives, especially those identified to be at risk of early cessation of EBF.

Author Contributions: Conceptualization, F.A.O.; data curation, F.A.O. and S.N.; formal analysis, F.A.O.; funding acquisition, F.A.O., J.U., J.P., B.B.A.M., and J.E.; investigation, F.A.O.; methodology, F.A.O.; project administration, F.A.O.; resources, F.A.O., A.M., A.P., and J.E.; software, F.A.O.; supervision, F.A.O.; validation, F.A.O., O.K.E., S.K., S.N., P.S., K.Y.A., A.M., O.O., K.A., A.P., B.B.A.M., and J.E.; visualization, F.A.O., O.K.E., S.N., P.S., K.Y.A., A.M., O.O., K.A., A.P., J.U., J.P., B.B.A.M., and J.E.; writing-original draft, F.A.O. and O.K.E.; writing-review and editing, F.A.O., O.K.E., S.K., S.N., P.S., K.Y.A., A.M., O.O., K.A., A.P., B.B.A.M., and J.E.

Funding: This research was funded by the early career research funding grant from the Office of the Deputy Vice-Chancellor (Research and Innovation), Western Sydney University, obtained by F.A.O. The APC was also funded through this grant.

Acknowledgments: The authors are grateful to all the health professionals in South Western Sydney Local Health Districts and Sydney Local Health District who spent time entering the data, and also to personnel (particularly Leslie Cramer for data download) at the Information Management \& Technology Division for the time spent in generating the data for the analysis. The authors are also grateful to the Early Years Research Group at the Ingham Institute, South Western Sydney Local Health District and the Healthy Home And Neighbourhood at the Sydney Local Health District for the initial funding. F.A.O. is also grateful to Melinda Wolfenden at THRI, Western Sydney University, for the time and effort in proofreading the grant application and excellent administrative support. The effort of Victoria Blight in securing the research grant is also acknowledged. 
Conflicts of Interest: The authors declare no conflict of interest. K.A. is a Guest Editor on the Special Issue "Breastfeeding: Short and Long-Term Benefits to Baby and Mother" for Nutrients but did not play any role in the peer-review and decision-making process for this manuscript. The funder had no role in the design of the study; in the collection, analyses, or interpretation of data; in the writing of the manuscript, or in the decision to publish the results.

\section{References}

1. World Health Organization. Indicators for assessing infant and young child feeding practices: Part 1: Definitions. In Proceedings of the Consensus Meeting, Washington, DC, USA, 6-8 November 2007.

2. Gartner, L.M.; Morton, J.; Lawrence, R.A.; Naylor, A.J.; O'Hare, D.; Schanler, R.J.; Eidelman, A.I. American academy of pediatrics section on breastfeeding: Breastfeeding and the use of human milk. Pediatrics 2005, 115, 496-506. [PubMed]

3. Karlsson, O.; Rodosthenous, R.S.; Jara, C.; Brennan, K.J.; Wright, R.O.; Baccarelli, A.A.; Wright, R.J. Detection of long non-coding RNAs in human breastmilk extracellular vesicles: Implications for early child development. Epigenetics 2016, 11, 721-729. [CrossRef] [PubMed]

4. Fujita, M.; Lo, Y.J.; Brindle, E. Nutritional, inflammatory, and ecological correlates of maternal retinol allocation to breast milk in agro-pastoral Ariaal communities of northern Kenya. Am. J. Hum. Biol. 2017, 29, e22961. [CrossRef] [PubMed]

5. Verduci, E.; Banderali, G.; Barberi, S.; Radaelli, G.; Lops, A.; Betti, F.; Riva, E.; Giovannini, M. Epigenetic effects of human breast milk. Nutrients 2014, 6, 1711-1724. [CrossRef] [PubMed]

6. Mannan, H. Early Infant Feeding of Formula or Solid Foods and Risk of Childhood Overweight or Obesity in a Socioeconomically Disadvantaged Region of Australia: A Longitudinal Cohort Analysis. Int. J. Environ. Res. Public Health 2018, 15, 1685. [CrossRef] [PubMed]

7. Victora, C.G.; Horta, B.L.; de Mola, C.L.; Quevedo, L.; Pinheiro, R.T.; Gigante, D.P.; Gonçalves, H.; Barros, F.C. Association between breastfeeding and intelligence, educational attainment, and income at 30 years of age: A prospective birth cohort study from Brazil. Lancet Glob. Health 2015, 3, e199-e205. [CrossRef]

8. Victora, C.G.; Bahl, R.; Barros, A.J.; França, G.V.; Horton, S.; Krasevec, J.; Murch, S.; Sankar, M.J.; Walker, N.; Rollins, N.C. Breastfeeding in the 21st century: Epidemiology, mechanisms, and lifelong effect. Lancet 2016, 387, 475-490. [CrossRef]

9. León-Cava, N.; Lutter, C.; Ross, J.; Martin, L. Quantifying the Benefits of Breastfeeding: A Summary of the Evidence; Pan American Health Organization: Washington, DC, USA, 2002.

10. Australian Bureau of Statistics. Culturally and Linguistically Diversity (CALD) Characteristics. Available online: https://www.abs.gov.au/ausstats/abs@.nsf/Lookup/by\%20Subject/4529.0.00.003 \{\}2014 \{\}Main\% 20Features \{\}Cultural\%20and\%20Linguistic\%20Diversity\%20(CALD)\%20Characteristics \{\}13 (accessed on 18 January 2019).

11. Brewer, R. Culturally and Linguistically Diverse Women in the Australian Capital Territory: Enablers and Barriers to Achieving Social Connectedness; Women's Centre for Health Matters: Canberra, Australia, 2009.

12. Adily, A.; Ward, J. Improving health among culturally diverse subgroups: An exploration of trade-offs and viewpoints among a regional population health workforce. Health Promot. J. Aust. 2005, 16, $207-212$. [CrossRef]

13. Ogbo, F.A.; Eastwood, J.; Hendry, A.; Jalaludin, B.; Agho, K.E.; Barnett, B.; Page, A. Determinants of antenatal depression and postnatal depression in Australia. BMC Psychiatry 2018, 18, 49. [CrossRef]

14. Aminisani, N.; Armstrong, B.K.; Canfell, K. Cervical cancer screening in Middle Eastern and Asian migrants to Australia: A record linkage study. Cancer Epidemiol. 2012, 36, e394-e400. [CrossRef]

15. Schmied, V.; Olley, H.; Burns, E.; Duff, M.; Dennis, C.-L.; Dahlen, H.G. Contradictions and conflict: A meta-ethnographic study of migrant women's experiences of breastfeeding in a new country. BMC Pregnancy Childbirth 2012, 12, 163. [CrossRef] [PubMed]

16. Gallegos, D.; Vicca, N.; Streiner, S. Breastfeeding beliefs and practices of African women living in Brisbane and Perth, Australia. Mater. Child Nutr. 2015, 11, 727-736. [CrossRef] [PubMed]

17. Joseph, J.; Brodribb, W.; Liamputtong, P. "Fitting-in Australia" as nurturers: Meta-synthesis on infant feeding experiences among immigrant women. Women Birth 2018. [CrossRef] [PubMed] 
18. Ogbo, F.A.; Eastwood, J.; Page, A.; Arora, A.; McKenzie, A.; Jalaludin, B.; Tennant, E.; Miller, E.; Kohlhoff, J.; Noble, J. Prevalence and determinants of cessation of exclusive breastfeeding in the early postnatal period in Sydney, Australia. Int. Breastfeed. J. 2017, 12, 16. [CrossRef] [PubMed]

19. Hauck, Y.L.; Fenwick, J.; Dhaliwal, S.S.; Butt, J. A Western Australian survey of breastfeeding initiation, prevalence and early cessation patterns. Mater. Child Health J. 2011, 15, 260-268. [CrossRef] [PubMed]

20. Amir, L.H.; Donath, S.M. Socioeconomic status and rates of breastfeeding in Australia: Evidence from three recent national health surveys. Med. J. Aust. 2008, 189, 254-256. [PubMed]

21. Forster, D.A.; McLachlan, H.L.; Lumley, J. Factors associated with breastfeeding at six months postpartum in a group of Australian women. Int. Breastfeed. J. 2006, 1, 18. [CrossRef] [PubMed]

22. Scott, J.; Landers, M.; Hughes, R.M.; Binns, C. Factors associated with breastfeeding at discharge and duration of breastfeeding. J. Paediatr. Child Health 2001, 37, 254-261. [CrossRef]

23. Craig, P.L.; Knight, J.; Comino, E.; Webster, V.; Pulver, L.J.; Harris, E. Initiation and duration of breastfeeding in an aboriginal community in south western Sydney. J. Hum. Lact. 2011, 27, 250-261. [CrossRef]

24. South Western Sydney Local Health District. Research Strategy for South Western Sydney Local Health District 2012-2021; South Western Sydney Local Health District: Sydney, Australia, 2012.

25. Sydney Local Health District. Planning. Available online: https://www.slhd.nsw.gov.au/planning/profiles. html (accessed on 22 June 2016).

26. National Health and Medical Research Council. Infant Feeding Guidelines; National Health and Medical Research Council: Canberra, Australia, 2012.

27. Bramson, L.; Lee, J.W.; Moore, E.; Montgomery, S.; Neish, C.; Bahjri, K.; Melcher, C.L. Effect of early skin-to-skin mother-Infant contact during the first $3 \mathrm{~h}$ following birth on exclusive breastfeeding during the maternity hospital stay. J. Hum. Lact. 2010, 26, 130-137. [CrossRef]

28. Crenshaw, J.T. Healthy birth practice\# 6: Keep mother and baby together-It's best for mother, baby, and breastfeeding. J. Perinat. Educ. 2014, 23, 211-217. [PubMed]

29. Moore, E.R.; Bergman, N.; Anderson, G.C.; Medley, N. Early skin-to-skin contact for mothers and their healthy newborn infants. Cochrane Database Syst. Rev. 2016. [CrossRef] [PubMed]

30. World Health Organization. Guideline: Protecting, Promoting and Supporting Breastfeeding in Facilities Providing Maternity and Newborn Services; CC BY-NC-SA 3.0 IGO; World Health Organization: Geneva, Switzerland, 2017.

31. Eastwood, J.; Ogbo, F.A.; Hendry, A.; Noble, J.; Page, A. Early Years Research Group. The impact of antenatal depression on perinatal outcomes in Australian women. PLoS ONE 2017, 12, e0169907. [CrossRef] [PubMed]

32. Australian Bureau of Statistics. Technical Paper: Socio-Economic Indexes for Areas (SEIFA) 2011; Commonwealth of Australia: Canberra, Australia, 2013.

33. New South Wales Health Department. Unless They're Asked: Routine Screening for Domestic Violence in NSW Health: An Evaluation of the Pilot Project; New South Wales Health: Sydney, Australia, 2001.

34. Binns, C.; Gilchrist, D.; Gracey, M.; Zhang, M.; Scott, J.; Lee, A. Factors associated with the initiation of breast-feeding by Aboriginal mothers in Perth. Public Health Nutr. 2004, 7, 857-861. [CrossRef] [PubMed]

35. Van Buuren, S.; Boshuizen, H.C.; Knook, D.L. Multiple imputation of missing blood pressure covariates in survival analysis. Stat. Med. 1999, 18, 681-694. [CrossRef]

36. Sterne, J.A.; White, I.R.; Carlin, J.B.; Spratt, M.; Royston, P.; Kenward, M.G.; Wood, A.M.; Carpenter, J.R. Multiple imputation for missing data in epidemiological and clinical research: Potential and pitfalls. BMJ 2009, 338, b2393. [CrossRef] [PubMed]

37. Spratt, M.; Carpenter, J.; Sterne, J.A.; Carlin, J.B.; Heron, J.; Henderson, J.; Tilling, K. Strategies for multiple imputation in longitudinal studies. Am. J. Epidemiol. 2010, 172, 478-487. [CrossRef]

38. Colaizy, T.T.; Saftlas, A.F.; Morriss, F.H. Maternal intention to breast-feed and breast-feeding outcomes in term and preterm infants: Pregnancy Risk Assessment Monitoring System (PRAMS), 2000-2003. Public Health Nutr. 2012, 15, 702-710. [CrossRef]

39. Wu, Y.; Ho, Y.; Han, J.; Chen, S. The influence of breastfeeding self-efficacy and breastfeeding intention on breastfeeding behavior in postpartum women. J. Nurs. 2018, 65, 42-50.

40. Stuebe, A.M.; Bonuck, K. What predicts intent to breastfeed exclusively? Breastfeeding knowledge, attitudes, and beliefs in a diverse urban population. Breastfeed. Med. 2011, 6, 413-420. [CrossRef]

41. Kools, E.J.; Thijs, C.; Vries, H.d. The behavioral determinants of breast-feeding in The Netherlands: Predictors for the initiation of breast-feeding. Health Educ. Behav. 2005, 32, 809-824. [CrossRef] [PubMed] 
42. Negin, J.; Coffman, J.; Vizintin, P.; Raynes-Greenow, C. The influence of grandmothers on breastfeeding rates: A systematic review. BMC Pregnancy Childbirth 2016, 16, 91. [CrossRef] [PubMed]

43. Cameron, A.J.; Hesketh, K.; Ball, K.; Crawford, D.; Campbell, K.J. Influence of peers on breastfeeding discontinuation among new parents: The Melbourne InFANT Program. Pediatrics 2010, 126, e601-e607. [CrossRef] [PubMed]

44. Shakya, P.; Kunieda, M.K.; Koyama, M.; Rai, S.S.; Miyaguchi, M.; Dhakal, S.; Sandy, S.; Sunguya, B.F.; Jimba, M. Effectiveness of community-based peer support for mothers to improve their breastfeeding practices: A systematic review and meta-analysis. PLoS ONE 2017, 12, e0177434. [CrossRef] [PubMed]

45. Susin, L.R.O.; Giugliani, E.R.J. Inclusion of fathers in an intervention to promote breastfeeding: Impact on breastfeeding rates. J. Hum. Lact. 2008, 24, 386-392. [CrossRef] [PubMed]

46. Tohotoa, J.; Maycock, B.; Hauck, Y.L.; Howat, P.; Burns, S.; Binns, C.W. Dads make a difference: An exploratory study of paternal support for breastfeeding in Perth, Western Australia. Int. Breastfeed. J. 2009, 4, 15. [CrossRef]

47. Maycock, B.; Binns, C.W.; Dhaliwal, S.; Tohotoa, J.; Hauck, Y.; Burns, S.; Howat, P. Education and support for fathers improves breastfeeding rates: A randomized controlled trial. J. Hum. Lact. 2013, 29, 484-490. [CrossRef]

48. Mitra, A.K.; Khoury, A.J.; Hinton, A.W.; Carothers, C. Predictors of breastfeeding intention among low-income women. Mater. Child Health J. 2004, 8, 65-70. [CrossRef]

49. McIntyre, E.; Hiller, J.E.; Turnbull, D. Determinants of infant feeding practices in a low socio-economic area: Identifying environmental barriers to breastfeeding. Aust. N. Z. J. Public Health 1999, 23, 207-209. [CrossRef]

50. Cooklin, A.R.; Canterford, L.; Strazdins, L.; Nicholson, J.M. Employment conditions and maternal postpartum mental health: Results from the Longitudinal Study of Australian Children. Arch. Women's Ment. Health 2011, 14, 217-225. [CrossRef]

51. New South Wales Health. Youth Health Resources and Contacts for Young People. Available online: https://www.health.nsw.gov.au/kidsfamilies/youth/Pages/yh-resources-for-young-people.aspx (accessed on 22 April 2019).

52. Grigoriadis, S.; VonderPorten, E.H.; Mamisashvili, L.; Tomlinson, G.; Dennis, C.-L.; Koren, G.; Steiner, M.; Mousmanis, P.; Cheung, A.; Radford, K. The impact of maternal depression during pregnancy on perinatal outcomes: A systematic review and meta-analysis. J. Clin. Psychiatry 2013, 74, 321-341. [CrossRef] [PubMed]

53. Dennis, C.-L.; McQueen, K. The relationship between infant-feeding outcomes and postpartum depression: A qualitative systematic review. Pediatrics 2009, 123, e736-e751. [CrossRef] [PubMed]

54. Rahman, A.; Hafeez, A.; Bilal, R.; Sikander, S.; Malik, A.; Minhas, F.; Tomenson, B.; Creed, F. The impact of perinatal depression on exclusive breastfeeding: A cohort study. Mater. Child Nutr. 2016, 12, 452-462. [CrossRef] [PubMed]

55. Beyondblue. Clinical practice guidelines for depression and related disorders-Anxiety, bipolar disorder and puerperal psychosis-In the perinatal period. In A Guideline for Primary Care Health Professionals; Beyondblue: Melbourne, Australia, 2011.

56. Buist, A.E.; Barnett, B.E.; Milgrom, J.; Pope, S.; Condon, J.T.; Ellwood, D.A.; Boyce, P.M.; Austin, M.-P.V.; Hayes, B.A. To screen or not to screen-that is the question in perinatal depression. Med. J. Aust. 2002, 177, S101-S105. [PubMed]

57. Kendall-Tackett, K.A. Violence against women and the perinatal period: The impact of lifetime violence and abuse on pregnancy, postpartum, and breastfeeding. Trauma Violence Abus. 2007, 8, 344-353. [CrossRef] [PubMed]

58. Martin-de-las-Heras, S.; Velasco, C.; Luna-del-Castillo, J.; Khan, K. Breastfeeding avoidance following psychological intimate partner violence during pregnancy: A cohort study and multivariate analysis. BJOG Int. J. Obstet. Gynaecol. 2018, 126, 778-783. [CrossRef] [PubMed]

59. Australian Institute of Health and Welfare. Screening for Domestic Violence during Pregnancy: Options for Future Reporting in the National Perinatal Data Collection; AIHW: Canberra, Australia, 2015.

60. Amir, L.H.; Donath, S.M. Does maternal smoking have a negative physiological effect on breastfeeding? The epidemiological evidence. Birth 2002, 29, 112-123. [CrossRef]

61. Amir, L.H. Maternal smoking and reduced duration of breastfeeding: A review of possible mechanisms. Early Hum. Dev. 2001, 64, 45-67. [CrossRef] 
62. Napierala, M.; Mazela, J.; Merritt, T.A.; Florek, E. Tobacco smoking and breastfeeding: Effect on the lactation process, breast milk composition and infant development. A critical review. Environ. Res. 2016, 151, 321-338. [CrossRef]

63. Australian National Preventive Health Agency. Smoking and Disadvantage; Australian National Preventive Health Agency: Canberra, Australia, 2013.

64. Smith, J.; Cattaneo, A.; Iellamo, A.; Javanparast, S.; Atchan, M.; Gribble, K.; Hartmann, B.; Salmon, L.; Tawia, S.; Hull, N.; et al. Review of Effective Strategies to Promote Breastfeeding: An. Evidence Check Rapid Review Brokered by the Sax Institute for the Department of Health; Sax Institute: Sydney, Australia, 2018.

65. Australian Government Department of Health. Breastfeeding. Available online: http://www.health.gov.au/ internet/main/publishing.nsf/Content/health-pubhlth-strateg-brfeed-index.htm (accessed on 22 April 2019).

(C) 2019 by the authors. Licensee MDPI, Basel, Switzerland. This article is an open access article distributed under the terms and conditions of the Creative Commons Attribution (CC BY) license (http://creativecommons.org/licenses/by/4.0/). 